\title{
Dynamics of Glucocorticoid and Mineralocorticoid Receptors in the Xenopus laevis Pituitary Pars Intermedia
}

\author{
Eric W. Roubos, ${ }^{a}$ Miyuki Kuribara, ${ }^{a}$ \\ Frouwke J. Kuipers-Kwant, ${ }^{a}$ Tony A. J. M. Coenen, ${ }^{a}$ \\ Kari H. A. Meijer, ${ }^{a}$ Peter M. J. M. Gruijsen, ${ }^{a}$ \\ and Robert J. Denver ${ }^{b}$
${ }^{a}$ Department of Cellular Animal Physiology, Donders Centre for Neuroscience, European Graduate School of Neuroscience, University of Nijmegen, Nijmegen, Netherlands
${ }^{b}$ Department of Molecular, Cellular and Developmental Biology, University of Michigan, Ann Arbor, Michigan, USA

\begin{abstract}
We showed the presence of glucocorticoid (GR) and mineralocorticoid (MR) receptors in different populations of Xenopus laevis melanotrope cells and revealed their downregulation (MR) and upregulation (GR) during dark background adaptation. Corticosterone did not affect short-term intracellular calcium dynamics and $\alpha$-melanophorestimulating hormone secretion, suggesting a role for GR and MR in long-term processes in the melanotropes.
\end{abstract}

Key words: glucocorticoid receptors; mineralocorticoid receptors; Xenopus laevis; melanotrope cells; background adaptation; corticosterone

\section{Introduction}

The melanotrope cells in the amphibian pituitary pars intermedia release $\alpha$-melanophore-stimulating hormone $(\alpha \mathrm{MSH})$, which darkens skin color. Various environmental factors control distinct melanotrope cell events, such as biosynthesis, processing, and release of $\alpha \mathrm{MSH} .{ }^{1,2}$ Recently, we showed the presence of glucocorticoid receptors (GRs) in the Xenopus laevis pars intermedia, suggesting a role for adrenal steroid hormones in the regulation of melanotrope cell. ${ }^{3}$ Here we extend this finding by (1) demonstrating the presence of GR and mineralocorticoid receptors (MRs) in the pars intermedia, (2) revealing their subcellular occurrence in melanotropes, and (3) showing that

\footnotetext{
Address for correspondence: Eric W. Roubos, Department of Cellular Animal Physiology, Donders Centre for Neuroscience, EURON, University of Nijmegen, P.O. Box 9010, 6500 GL Nijmegen, the Netherlands. roubos@science.ru.nl
}

a dark background decreases the amount of GR and increases the amount of MR. Moreover, we studied a possible action of corticosterone (CORT) on the secretory activity (intracellular calcium dynamics and $\alpha \mathrm{MSH}$ secretion) of melanotrope cells.

\section{Materials and Methods}

Six-month-old $X$. laevis were placed on a white or a black background for 3 weeks. Paraffin sections $(7 \mu \mathrm{m})$ of Bouin-fixed brains were immunostained with our affinity-purified rabbit anti-X. laevis GR (1:25; see Ref. 3) or MR2 (1:50) sera. The MR2 serum was generated in a rabbit against two peptides (both conjugated to keyhole limpet hemocyanin) that correspond to positions 32-49 and 160-177 of the $X$. laevis MR. The MR sequences that we chose show no sequence similarity to other known frog steroid hormone receptors, and basic local alignment search tool (BLAST) search of 
the National Center for Biotechnology Information (NCBI) database showed no sequence similarity to other known proteins. We purified the antiserum over an Affi-Gel Protein A column, followed by affinity purification over a peptide affinity column with the MR peptides conjugated to Ultralink iodoacetyl matrix (Pierce Biotechnology Inc., Rockfold, IL), following the manufacturer's instructions. Immune complexes were detected using indocarbocyanine (Cy3)-coupled donkey anti-rabbit IgG (1:100) coupled with the tyramide signal amplification method. ${ }^{4}$ For immunodouble labeling, sections were stained with GR as described above and then incubated in, consecutively, $2 \% \mathrm{H}_{2} \mathrm{O}_{2}$ in $0.1 \mathrm{~mol} \mathrm{PBS}$ for $15 \mathrm{~min}$, anti-MR2 (1:50) for $16 \mathrm{~h}$, fluorescein isothiocyanate (FITC)-conjugated secondary donkey anti-rabbit serum for $3 \mathrm{~h}$, sheep antiFITC conjugated to horseradish peroxidase (1:100) for $1 \mathrm{~h}$, and fluorescein-conjugated tyramide solution $(1: 200)$ for $30 \mathrm{~min}$. For immuno-electron microscopy, ultrathin sections of high-pressure-frozen pars intermedia were treated with GR or MR antisera and incubated with $6 \mathrm{~nm}$ gold-conjugated, secondary goat-anti-rabbit serum. ${ }^{5}$ Immunostaining was absent when these sera had been omitted or when preabsorbed with immunizing peptides (GR, see Ref. 3; MR, M. Yao and R.J. Denver, unpublished data). For morphometry of six white- and six black-adapted animals, in the largest section of the pars intermedia GR- and MR-immunoreactive (ir) melanotrope cells were counted and their specific immunosignal density per cell (SSD) assessed, using Image J software (version 1.37; NIH, Bethesda, MD). For dynamic video imaging of intracellular calcium, single melanotropes of two black-adapted animals were loaded with fura-2 and microscopically analyzed using Metafluor v.4.5 software (Universal Imaging Corporation, Dowingtown, PA). CORT (100 $\mathrm{nmol} / \mathrm{L}$ ), in Xenopus Ringer's solution containing $0.000067 \%$ ethanol $\mathrm{v} / \mathrm{v}$, was applied for 30 min. Radioactive $\left({ }^{3} \mathrm{H}\right.$-lysine-labeled) peptide release was assessed during in vitro super-
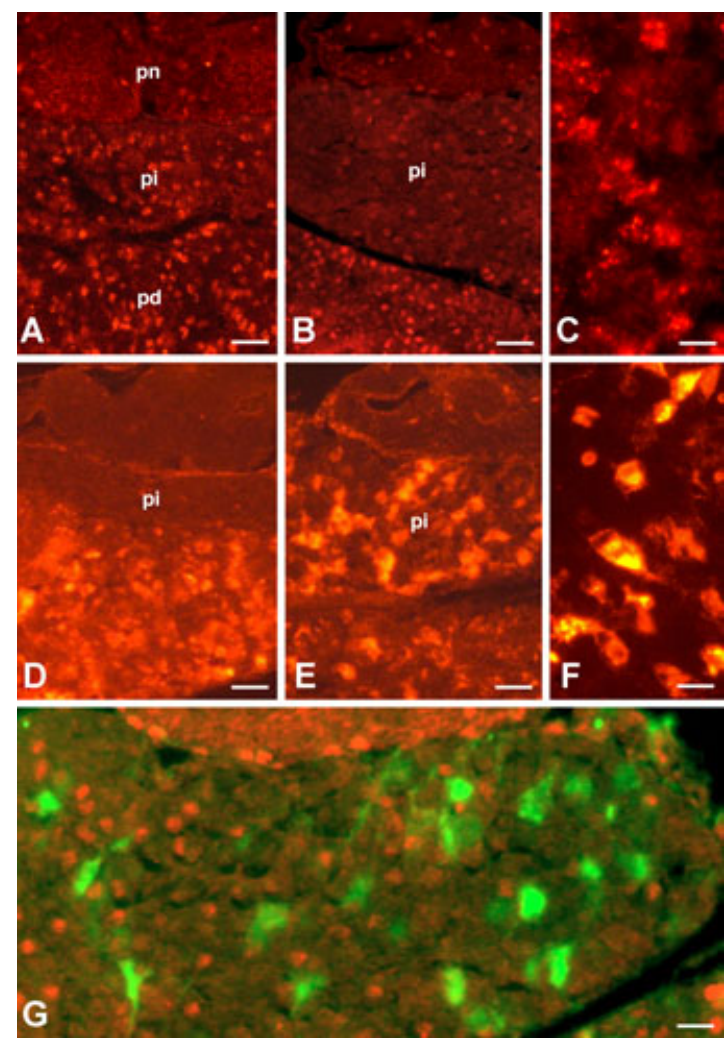

Figure 1. Immunocyłochemistry of (A-C) glucocorticoid receptors (GR) and (D-F) mineralocorticoid receptors (MR) in the pituitary gland of Xenopus laevis adapted to $(\mathbf{A}, \mathbf{C}, \mathbf{D})$ a white or to $(\mathbf{B}, \mathbf{E}, \mathbf{F}, \mathbf{G})$ a black background. (G) Double labeling showing separate melanotrope cell populations for GR (indocarbocyanine; red) and MR (fluorescein isothiocyanate; green). pd, pars distalis; pi, pars intermedia; pn, pars nervosa. Bars $(\mathbf{A}, \mathbf{B}, \mathbf{D}, \mathbf{E}) 25 \mu \mathrm{m}$, bars $(\mathbf{C}, \mathbf{F}, \mathbf{G})$ $10 \mu \mathrm{m}$. (In color in Annals online.)

fusion. Data were tested for homogeneity of variance and normality and then entered into analysis of variance $(\alpha=5 \%)$. All experiments were performed in accordance with the Dutch law concerning animal welfare.

\section{Results and Discussion}

In the pars intermedia the majority of melanotrope cells showed GR-ir, especially in their nucleus (Fig. 1A-C). With anti-MR a substantial number of melanotropes, dispersed over the lobe, revealed labeling in the cytoplasm 

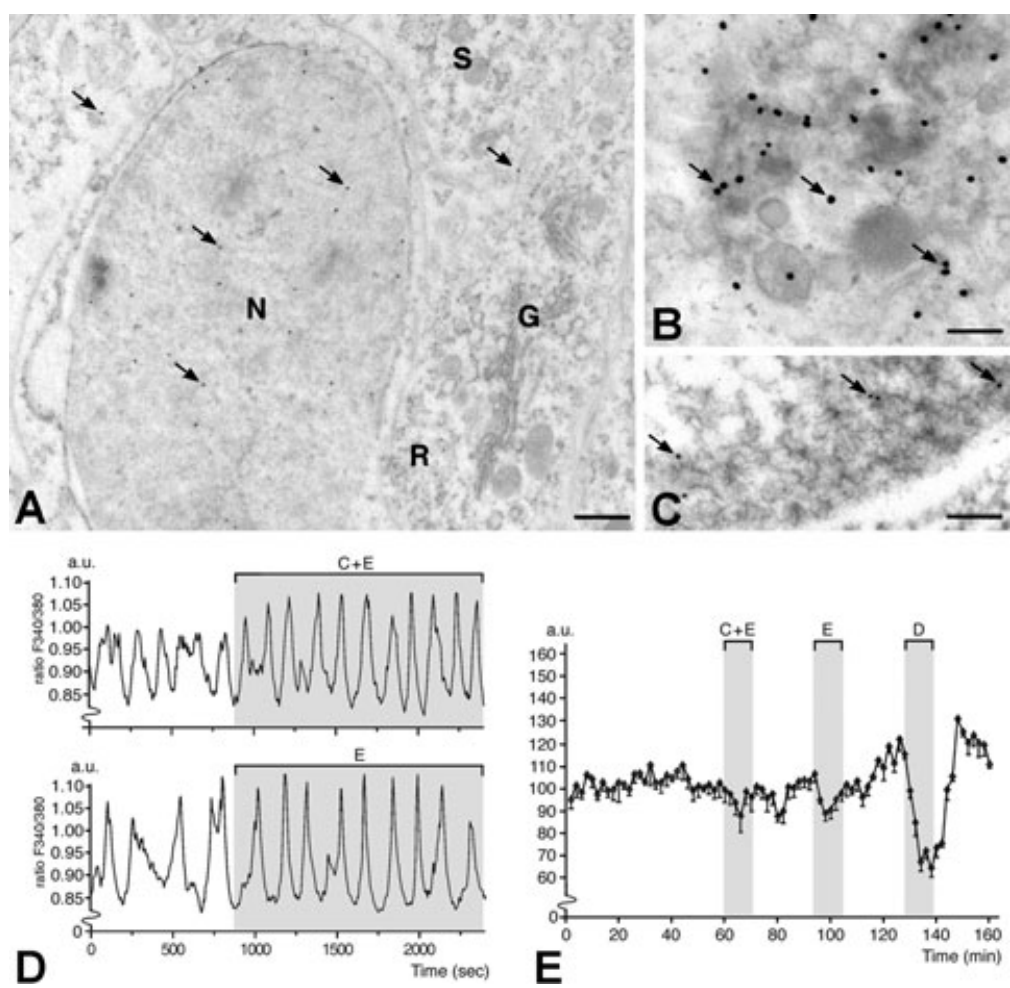

Figure 2. Melanotrope cells of $X$. laevis. Immunogold (arrows) electron microscopy shows (A) GR-immunoreactive (ir) in the cell nucleus and (B) MR-ir in tubule- and vesicle-rich and (C) in nuclear chromatin-rich areas. Corticosterone $\left(C ; 10^{-7}\right.$ mol) does not affect (D) melanotrope calcium oscillations (dynamic video imaging) or (E) radioactive peptide release (in vitro superfusion). D, $10^{-6} \mathrm{~mol}$ dopamine; $E$, ethanol; $G$, Golgi apparatus; $N$, nucleus; $R$, rough endoplasmic reticulum; S, secretory granule. Bar (A) $500 \mathrm{~nm}$, bars (B,C) $100 \mathrm{~nm}$.

and, particularly, in the nucleoplasm (Fig. 1DF). Immunodouble labeling demonstrated that GR-ir and MR-ir generally did not coexist within the same cells (Fig. 1G). Whether this differential steroid receptor presence is related to functional heterogeneity of the amphibian pars intermedia deserves further research. Immunoelectron microscopy showed immunogold particles especially in tubule and vesicle-rich areas in the cytoplasm and in chromatin-rich areas in the nucleus (Fig. 2A-C). To study if the presence of GR and MR depends on the activity state of the melanotrope cells, we quantified their immunolabeling in animals on a white and a black background. The pars intermedia of black-adapted animals had a much larger thickness (2.5 times; $P<0.001)$ and volume (3.7 times; $P<0.001)$ than that of white-adapted ones. In the latter, GR-ir cells had a similar
SSD as in black ones, but their number per unit of surface area of the pars intermedia was about seven times higher $(P<0.0001)$. Therefore, it seems that the total amount of GR in the pars intermedia is upregulated by white adaptation. In contrast to GR, MR were upregulated in black animals, with a higher SSD $(+30 \%$; $P<0.05)$ and a much greater number of MRir cells $(12.1$ times; $P<0.0001)$ than in white animals.

When isolated melanotrope cells were perfused in vitro with $10^{-7}$ mol CORT, the frequency and amplitude of spontaneous calcium oscillations were slightly increased, but this effect was a result of the solvent (Fig. 2D). Also, no effect of CORT on radiolabeled peptide release was seen (Fig. 2E). Therefore, CORT does not influence calcium oscillation-driven secretion of $\alpha \mathrm{MSH}$. Possibly (adrenal) steroids act upon 
$\mathrm{MR}$ and/or GR receptors to change long-term processes in the melanotrope cell, such as expression of the $\alpha \mathrm{MSH}$ precursor protein proopiomelanocortin. ${ }^{4}$

We propose that the Xenopus melanotrope cell exemplifies how the adrenal gland can set long-term sensitivity of a behavior-controlling endocrine cell to external physiological stimuli, depending on the animal's physiological (stress) condition.

\section{Conflicts of Interest}

The authors declare no conflicts of interest.

\section{References}

1. Roubos, E.W., W.J.J.M. Scheenen \& B.G. Jenks. 2005. Neuronal, neurohormonal, and autocrine control of
Xenopus melanotrope cell activity. In Trends in Comparative Endocrinology and Neurobiology, Vol. 1040. H. Vaudry, E.W. Roubos, L. Schoofs, et al., Eds.: 172-183. Annals N.Y. Academy of Sciences. New York.

2. Jenks, B.G., A.H. Kidane, W.J.J.M. Scheenen, et al. 2007. Plasticity in the melanotrope neuroendocrine interface of Xenopus laevis. Neuroendocrinology 994: $1-9$.

3. Yao, M., F. Hu \& R.J. Denver. 2008. Distribution and corticosteroid regulation of glucocorticoid receptor in the brain of Xenopus laevis. F. Comp. Neurol. 508: 967982.

4. Kidane, A.H., E.W. Roubos \& B.G. Jenks. 2008. Pituitary adenylate cyclase-activating polypeptide regulates brain-derived neurotrophic factor exon IV expression through the VPAC1 receptor in the amphibian melanotrope cell. Endocrinology 149: 4177-4182.

5. Roubos, E.W., G. Lázár, M. Calle, et al. 2008. Brain distribution and evidence for both central and neurohormonal actions of cocaine- and amphetamineregulated transcript peptide in Xenopus laevis. F. Comp. Neurol. 507: 1622-1638. 